\title{
Energy Dissipation, Runoff Production, and the Three-Dimensional Structure of River Basins
}

\author{
Ignacio Rodríguez-Iturbe, ${ }^{1,2}$ ANDrea Rinaldo, ${ }^{3}$ RicCardo Rigon, ${ }^{4}$ \\ Rafael L. Bras, ${ }^{2}$ Alessandro Marani, ${ }^{4}$ AND Ede IJjÁsz-VÁsQUeZ ${ }^{2}$
}

\begin{abstract}
Three principles of optimal energy expenditure are used to derive the most important structural characteristics observed in drainage networks: (1) the principle of minimum energy expenditure in any link of the network, (2) the principle of equal energy expenditure per unit area of channel anywhere in the network, and (3) the principle of minimum total energy expenditure in the network as a whole. Their joint application results in a unified picture of the most important empirical facts which have been observed in the dynamics of the network and its three-dimensional structure. They also link the process of runoff production in the basin with the characteristics of the network.
\end{abstract}

\section{InTRoduction: The ConNectivity Issue}

Well-developed river basins are made up of two interrelated systems: the channel network and the hillslopes. The hillslopes control the production of runoff which in turn is transported through the channel network toward the basin outlet. Every branch of the network is linked to a downstream branch for the transportation of water and sediment but it is also linked for its viability, through the hillslope system, to every other branch in the basin. Hillslopes are the runoff-producing elements which the network connects, transforming the spatially distributed potential energy arising from rainfall in the hillslopes to kinetic energy in the flow through the channel reaches. In this paper we focus on the drainage network as it is controlled by energy dissipation principles. It is precisely the need for effective connectivity that leads to the treelike structure of the drainage network. Figure 1, from Stevens [1974], illustrates this point. Assume one wishes to connect a set of points in a plane to a common outlet and for illustration purposes assume that every point is equally distant from its nearest neighbors. Two extreme ways to establish the connection would be through the spiral and the explosion type of patterns. The explosion pattern has the advantage that it connects every parcel of the system to the outlet in the most direct manner. Nevertheless it rejects any kind of interaction between the different parts and the total path length for the system as a whole is extremely large. Thus although it has the minimum average path connecting each parcel to the outlet, it lacks shortness as a whole. The spiral pattern on the other hand is quite short for the systems as a whole, but it leads to an extremely large average path from a point to the outlet. One is tempted to say that from an organizational point of view the spiral represents pure socialism and the explosion pure capitalism. In one case the system is supposed to operate at its best as a whole with a total disregard for the average individual, who finds himself in the worst possible condition. In the other

\footnotetext{
${ }^{1}$ Instituto Internacional de Estudios Avanzados, Caracas.

${ }^{2}$ Ralph M. Parsons Laboratory, Massachusetts Institute of Technology, Cambridge.

${ }^{3}$ Universita di Trento, Trento, Italy.

${ }^{4}$ Universita di Venezia, Venice, Italy.

Copyright 1992 by the American Geophysical Union.

Paper number 91WR03034.

0043-1397/92/91WR-03034\$05.00
}

case each individual is supposed to operate at his best completely oblivious of his neighbors, but the system as a whole cannot survive.

Branching patterns accomplish connectivity combining the best of the two extremes; they are short as well as direct. The drainage network, as well as many other natural connecting patterns, is basically a transportation system for which the treelike structure is a most appealing structure from the point of view of efficiency in the construction, operation and maintenance of the system.

The drainage network accomplishes connectivity for transportation in three dimensions working against a resistance force derived from the friction of the flow with the bottom and banks of the channels, the resistance force being itself a function of the flow and the channel characteristics. This makes the analysis of the optimal connectivity a complex problem that cannot be separated from the individual optimal channel configuration and from the spatial characterization of the runoff production inside the basin. The question is whether there are general principles that relate the structure of the network and its individual elements with the rate of energy expenditure which takes place in the system as a whole and in each of its elements.

\section{Principles of Energy Expenditure In Drainage Networks}

A link of a drainage network carries a wide range of discharges resulting from a variety of rainfall events (of different intensities and duration) and antecedent conditions of soil moisture. The individual channel characteristics are commonly assumed to be controlled by the bank-full discharge that the channel is capable of transporting. It is also true, though, that most of the work the flow performs throughout time takes place at discharges smaller than the bank-full capacity. From this point of view the mean annual flow may be considered a more representative discharge condition to characterize the work being done by the flow. Thus it is likely that any principles of optimal energy expenditure responsible for the three-dimensional structure of the drainage network will yield the same type of results when applied to the case of bank-full discharges as when the flow is characterized at every link by the corresponding mean annual value.

Three different principles are now postulated: (1) the principle of minimum energy expenditure in any link of the 

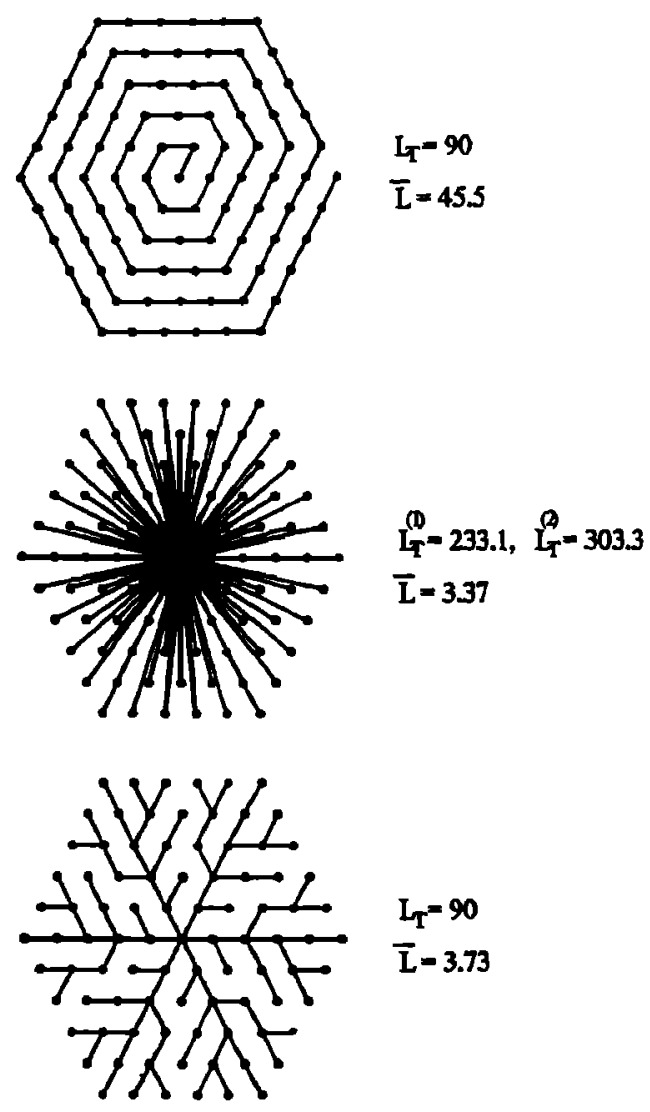

Fig. 1. Different patterns of connectivity of a set of equally spaced points to a common outlet. $L_{T}$ is the total length of the paths, and $\bar{L}$ is the average length of the path from a point to the outlet. In the explosion case, $L_{f}^{(2)}$ refers to the case when there is a minimum displacement among the points so that there is a different path between each point and the outlet [from Stevens, 1974].

network; (2) the principle of equal energy expenditure per unit area of channel anywhere in the network; and (3) the principle of minimum energy expenditure in the network as a whole. It will be shown that the combination of these principles is a sufficient explanation for the treelike structure of the drainage network and, moreover, that they explain many of the most important empirical relationships observed in the internal organization of the network and its linkage with the flow characteristics. The first principle expresses a local optimal condition for any link of the network. The second principle expresses an optimal condition throughout the network regardless of its topological structure and later on in this paper will be interpreted in a probabilistic framework. It postulates that energy expenditure is the same everywhere in the network when normalized by the area of the channel on which it takes place. Thus even with the first principle there will be channels that spend much more energy per unit time than others only because of their larger discharge. The second principle makes all channels equally efficient when adjusted for size. The third principle is addressed to the topological structure of the network and refers to the optimal arrangement of its elements.

The first principle is similar to the principle of minimum work used in the derivation of Murray's law in physiological vascular systems. Murray [1926] derived a relation which states that the cube of the radius of a parent vessel should equal the sum of the cubes of the radii of the daughter vessels (see, for example, Sherman [1981]). He assumed that two energy terms contribute to the cost of maintaining blood flow in any vessel: (1) the energy required to overcome friction as described by Poiseuille's law, and (2) the energy metabolically involved in the maintenance of the blood volume and vessel tissue. Minimization of the cost function leads to the radius of the vessel being proportional to the $1 / 3$ power of the flow. Uylings [1977] has shown that when turbulent flow is assumed in the vessel, rather than laminar conditions, the same approach leads to the radius being proportional to the $3 / 7$ power of the flow. The second principle was conceptually suggested by Leopold and Langbein [1962] in their studies of landscape evolution. It is of interest to add that minimum rate of work principles have been applied in several contexts in geomorphic research. Optimal junction angles have been studied in this context by Howard [1971], Roy [1983], and Woldenberg and Horsfield [1986], among others. Also the concept of minimum work as a criterion for the development of stream networks has been discussed under different perspectives by Yang [1971] and Howard [1990], among others.

\section{ENERgY EXPENDITURE AND OPTIMAL NETWORK CONFIgURATION}

Consider a channel of width $w$, length $L$, slope $S$, and flow depth $d$. The force responsible for the flow is the downslope component of the weight, $F_{1}=\rho g d L w \sin \beta=\rho g d L w S$ where $\sin \beta \simeq \tan \beta=S$. The force resisting the movement is the stress per unit area times the wetted perimeter area, $F_{2}=\tau(2 d+w) L$, where a rectangular section has been assumed in the channel. Under conditions of no acceleration of the flow, $F_{1}=F_{2}$, and then $\tau=\rho g S R$ where $R$ is the hydraulic radius $R=A_{w} / P_{w}=w d /(2 d+w), A_{w}$ and $P_{w}$ being the cross-sectional flow area, and the wetted perimeter of the section, respectively. In turbulent incompressible flow the boundary shear stress varies proportionally to the square of the average velocity, $\tau=C_{f} \rho v^{2}$, where $C_{f}$ is a dimersionless resistance coefficient. Equating the two expressions for $\tau$, one obtains the well-known relationship, $S=C_{f} v^{2}$ $(R g)$, which gives the losses due to friction per unit weight of flow per unit length of channel. There is also an expenditure of energy related to the maintenance of the channel which may be represented by $F$ (soil, flow) $P_{w} L$ where $F(\quad)$ is a complicated function of soil and flow properties representine the work per unit time and unit area of channel involved in the removal and transportation of the sediment which otherwise would accumulate in the channel surface. From the equations of bed load transport one may assume that $F=$ $K \tau^{m}$ where $K$ depends only on the soil and fluid properties and $m$ is a constant.

In a channel of length $L$ and flow $Q$ the rate of enengy expenditure may then be written as

$$
\begin{aligned}
P=C_{f} \rho \frac{v^{2}}{R} Q L+K \tau^{m} P_{w} L= & C_{f} \rho P_{w} \frac{Q^{3}}{A_{w}^{3}} L \\
& +K C_{f}^{m} \rho^{m} v^{2 m} P_{w} L
\end{aligned}
$$

The coefficient $C_{f}$ depends mainly on the channel roughness which tends to decrease only slightly in the downstrean direction; on the whole the downstream reduction in rougl 
ness resulting from a decrease in particle size is compensated by other forms of flow resistance like that offered by bars and channel bends [Leopold et al., 1964]. According to the second principle of energy expenditure, $P_{1}=P /\left(P_{w} L\right)$ is the same anywhere in the network. Substituting $P$ from (1), one obtains

$$
P_{1}=C_{f} \rho v^{3}+K C_{f}^{m} \rho^{m} v^{2 m}=\text { const }
$$

which implies that the velocity tends to be constant throughout the network. This has been corroborated by the field investigations of Leopold and Maddock [1953], Wolman [1955], and Brush [1961], who obtained values of $z<0.1$ in the downstream relation between velocity and discharge, $v=C Q^{z}$, this being the case for both mean annual flow conditions or bank-full discharges throughout the network. Also the field experiments of Pilgrim [1977] corroborate this finding, although as pointed out by Howard [1990], this may not be a universal kind of behavior. Substituting the width $w=Q /(v d)$ in (1) one gets:

$$
\begin{aligned}
P=\frac{Q L}{d}\left[C_{f} \rho v^{2}+\right. & \left.K C_{f}^{m} \rho^{m} v^{2 m-1}\right] \\
& +d L\left[2 C_{f} \rho v^{3}+2 K C_{f}^{m} \rho^{m} v^{2 m}\right]
\end{aligned}
$$

the terms in brackets being constant throughout the network for a given flow condition. According to the first principle of energy expenditure, $P$ should be a minimum in any link of the network. If the link is transporting a discharge $Q$, this means $d P / d(d)=0$ which yields

$$
Q=\text { (const) } d^{2} \text { or } d=\text { (const) } Q^{0.5}
$$

Thus in any link of a network the mean annual flow or the bank-full discharge is proportional to the square of their corresponding flow depths, the constant of proportionality being the same everywhere in the network. The above result has been observed by field investigators. Leopold et al. [1964] found $d \sim Q^{f}$ with $f=0.4$ for the dependence of depth on flow in the downstream direction, with the same exponent valid both for bank-full conditions and for mean annual flow conditions. Using (4) in the expression for width, $w=Q /(v d)$, gives

$$
w=\text { (const) } Q^{0.5}
$$

which says that in the downstream direction the width varies proportionally to the square root of the discharge. Leopold et al. [1964] found a very good relationship between width and the square root of the discharge in the downstream direction for both bank-full and mean annual flow conditions.

Substituting (4) in (3) we obtain the optimal power expenditure at any link as

$$
P=k Q^{0.5} L
$$

Adding over all links of the network we obtain the total rate of energy expenditure under optimal conditions:

$$
E=\sum_{i} P_{i}=k \sum_{i} Q_{i}^{0.5} L_{i}
$$

where $k$ varies with the discharge but is constant throughout the network if mean annual flow or bank-full conditions are operating throughout the basin. In an explosion pattern like the one of Figure 1 , the $Q_{i}$ are small since there is no aggregation of flows from tributary links; on the other hand, the sum of the $L_{i}$ is extremely large and so is $E$. If each node in Figure 1 has a constant discharge, $Q$, then

$$
E=k Q^{0.5} L_{T}
$$

where $L_{T}$ is the total path length. In the case of the spiral pattern with a constant discharge at every node, one has

$$
E=k L Q^{0.5}\left[1+2^{0.5}+3^{0.5}+\cdots+N^{0.5}\right]
$$

where $L$ is the constant distance between neighboring points. Although $L_{T}$ is small for the spiral, $E$ is again prohibitively large. On the other hand, the treelike pattern combines a piecewise aggregation of flows throughout the system at the same time that it keeps quite short the total length of the flow paths. This yields a much smaller total rate of energy expenditure, $E$. In the case of Figure 1 if the input flow at any node is taken as equal to 1 , the corresponding values of $E$ are as follows: spiral, $574 k$; explosion, $303 k$; and treelike, $151 k$. The explosion pattern is only relatively competitive when most of the points are close to the common outlet. If one keeps adding points further away from the outlet, the total length of the explosion pattern increases dramatically and so does the total energy expenditure, $E$. The above comparison although illustrative is only correct if one assumes that $k$ is the same in all cases, which implies that the flow velocity has remained the same in all cases, which is not necessarily true in natural networks.

It is interesting to compare networks with different topological structure which have the same total length, drain the same total area and carry the same total flow. One could think of two criteria to make this particular comparison. One is the criterion of minimum total energy expenditure $E$, and the other is the criterion of minimum energy expenditure per unit area of channel, $P_{1}$, which remains constant throughout each network. These two criteria oppose each other in the sense that when $P_{1}$ is the same among different topological arrangements, then the minimum $E$ corresponds to the network with the highest degree of branching; but when $E$ is taken constant for the various networks, then the minimum $\boldsymbol{P}_{1}$ corresponds to the network with the smallest degree of branching. This is the subject of the next section of this paper.

\section{Horton's Laws and Optimal ENERgy EXPENDITURE}

The fantastic variety of forms and shapes of drainage networks embodies a deep sense of regularity in formal relations among the parts, an important example of which are the empirical laws found by Horton [1945]. Horton's law of stream numbers involves the relative arrangement of streams which he stated as

the number of streams of different orders in any given drainage basin tends closely to approximate an inverse geometric series in which the first term is unity and the ratio is the bifurcation ratio [Horton, 1945, p. 291].

Mathematically it is expressed as 


$$
\frac{N_{\omega}}{N_{\omega+1}}=R_{b}
$$

where $N_{\omega}$ is the number of streams of order $\omega$, and $R_{b}$ is the bifurcation ratio. $N_{\omega}$ is usually estimated with the Strahler ordering procedure: (1) channels that originate at a source are defined as first-order streams; (2) when two streams of order $\omega$ join, a stream of order $\omega+1$ is created; and (3) when two streams of different order join, the channel segment immediately downstream has the higher order of the two combining streams. $N_{\Omega}=1$, where $\Omega$ is the order of the basin network which for a fixed number of sources, $N_{1}$, is a measure of the degree of branching. Horton's law of stream lengths is expressed as

$$
\frac{\bar{L}_{\omega}+1}{\bar{L}_{\omega}}=R_{L}
$$

where $\bar{L}_{\omega}$ is the average length of streams of order $\omega$ and $R_{L}$ is the length ratio.

Shreve [1966] provided a statistical interpretation of Horton's law of stream numbers. He defined a topologically random population of channel networks as a population within which all topologically distinct networks with a given number of links, or equivalently with a given number of sources, are equally likely. Topologically distinct networks are those whose schematic map projections cannot be continuously deformed and rotated in the plane of projection so as to become congruent. Shreve [1966] proposed that in the absence of geological controls a natural population of channel networks will be topologically random. He noticed that inherent in the definition of stream order is the corollary that no arborescent network can depart very far from Horton's geometric series law. The fact that for every stream of given order, except the first, there must be at least two streams of the next lower order means that on a Horton diagram of log $N_{\omega}$ versus $\omega$, the points for any channel network with given order and given number of sources "will necessarily lie within a relatively restricted parallelogram-shaped region whose long diagonal is the locus of points which exactly satisfy Horton's law" [Shreve, 1966, p. 121]. Moreover it is around the diagonal of the narrow parallelogram where most of the networks with given order and number of sources will be located. Thus the fact that natural networks tend to fulfill Horton's law of stream numbers is really a consequence of the ordering system. Most intriguing is the fact that drainage networks tend to have a bifurcation ratio, $R_{b}$, close to 4 with most values lying in a small range between 3 and 5 . Shreve showed that in a topologically random population of networks with a given number of sources, the most probable network order $\Omega$ is that which makes $R_{b}$ closest to 4 .

Shreve [1969] provided a statistical interpretation of Horton's law of stream lengths. Taking the link lengths as random variables with a common distribution he showed that networks tend to follow Horton's law of stream lengths, the most probable networks giving the straightest lines in the plots of $\log \bar{L}_{\omega}$ versus $\omega$ with an $R_{L}$ of approximately 2 . This agrees well with the values observed in nature which lie between 1.5 and 3.5. La Barbera and Rosso [1987] and Tarboton et al. [1988] have shown that $R_{b}$ and $R_{L}$ are connected through the fact that drainage networks exhibit a fractal structure with fractal dimension given by

$$
D=\frac{\log R_{b}}{\log R_{L}}
$$

The measurements of Tarboton et al. [1988] indicate that these networks are space filling with $D=2$, which in turn implies $R_{b}=R_{L}^{2}$.

After Shreve's [1966, 1969] classical papers it has been commonly assumed by hydrologists and geomorphologists that the topological arrangement and relative sizes of the streams of a drainage network are just the result of a most probable configuration in a random environment. Thus the value of $R_{b}=4$ and the implied $R_{L}=2$ are explained solety as being those with the highest probability of occurrence. We believe that in an evolutionary system like the drainage network both chance and necessity should be operating: and, moreover, that the influence of necessity is felt through a tendency to minimize the total rate of energy expenditure in the network and the rate of energy expenditure per unit area of channel anywhere in the network. This will be shown through an example but the implications are general.

Figure 2 shows three networks with $N_{1}=16$ which exactly fulfill Horton's law of stream numbers. All three networks will be analyzed by two different sets of conservation rules. In case 1 they have been built with links of unit length and unit runoff production per link in all networks. In case 2 they all have the same total length and total area but each link is assigned an area draining directly into it equal to the square of its length as is commonly observed in drainage networks. The runoff produced directly at every link is considered equal to the area draining directly to the link. In case 2 with $R_{b}=4, R_{L}$ is fixed at 2 , and the total area is 80 , from which all the link lengths and their respective areas are then deduced. For case 2 with $R_{b}=2$ and $R_{b}=16$ the link lengths and the $R_{L}$ values are deduced by imposing the condition that the total area and total length should match those of the network with $R_{b}=4$. If the rate of energy expenditure per unit area of channel is maintained as the same in the three networks, implying that the flow velocity is the same in all of them, then the minimum total rate of energy expenditure, $E$, corresponds to the network with the largest $\Omega$ or $R_{b}=2$. The topological arrangement with largest total rate of energy expenditure is the one with the smallest $\Omega$ or $R_{b}=16$. This is always the case regardless of the number of sources. If one maintains the total rate of energy expenditure, $E$, as a constant in all cases, then the flow velocities need to be different, the largest velocity corresponding to $R_{b}=2$ and the smallest one to $R_{b}=16$. This, in turn, implies a rate of energy expenditure per unit area of channel, $\boldsymbol{P}_{1}$, largest for the network with maximum $\Omega$ and smallest for the network with the minimum $\Omega$. Again this will always be the case regardless of the number of sources. For $N_{\mathrm{l}}=16$ there is a very large number of topologically different networks which may have $\Omega$ values between 2 and 5 . The networks with $\Omega=3$ and $\Omega=4$ combine the best of two worlds; they try to be efficient from the point of view of the energy expenditure of the whole system but at the same time they try to maintain the rate of energy expenditure per unit area of channel small anywhere in the system. These networks are the ones with $R_{b}$ in the range of values observed in nature.

The above particular comparison among networks which have the same total length, drain the same total area and carry the same total flow assumes there are no restrictions in 

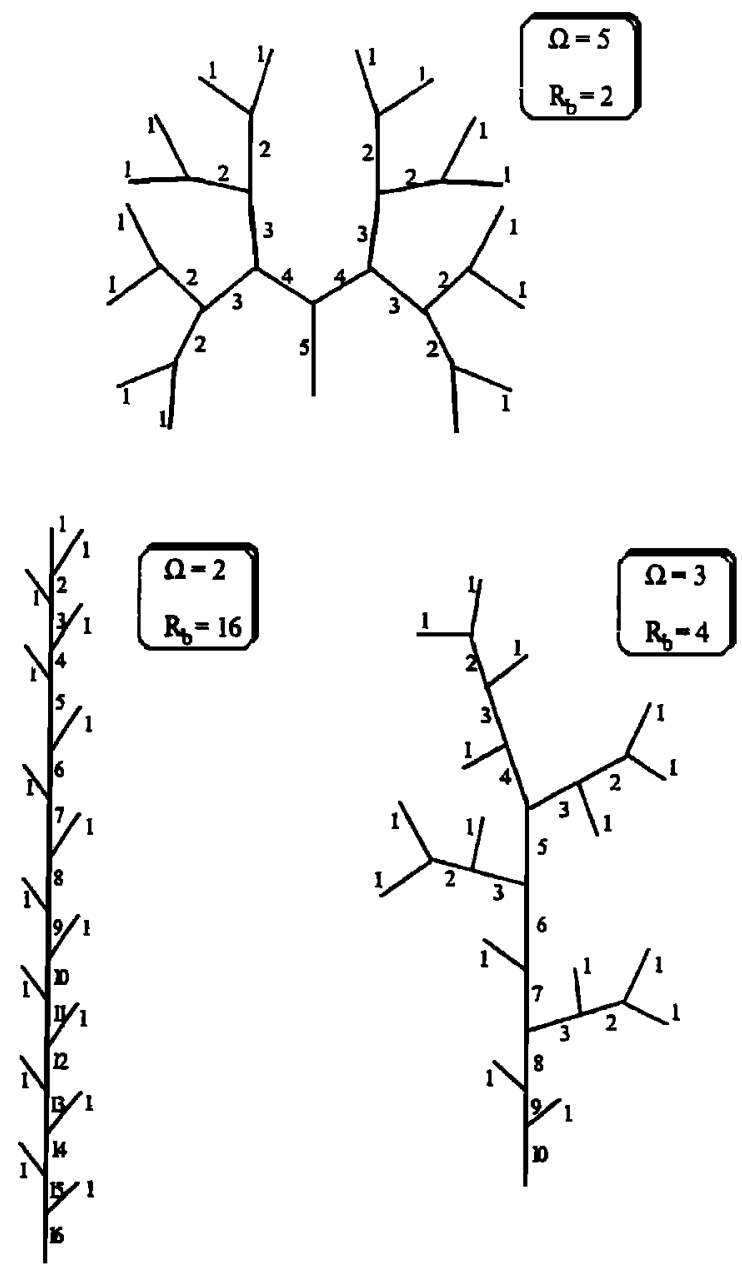

Fig. 2. Networks analyzed according to two sets of conservation rules. In case 1, all links have unit length in all networks. The discharges are as follows (where the numbers in parentheses correspond to those marked on the branches): for $\Omega=5$ : (1) 1 , (2) 3, (3) 7 , (4) 15 , (5) 31 ; for $\Omega=2$ : (1) 1 , (2) 3 , (3) $5, \cdots$, (15) 30 , (16) 31 ; for $\Omega=3$ : (1) 1 , (2) 3 , (3) 5, (4) 7, (5) 13 , (6) 19 , (7) 21, (8) 27 , (9) 29 , (10) 31. Total rates of energy expenditures are as follows: $\Omega=5$, $53.75 k ; \Omega=2,75.42 k ; \Omega=3,63.2 k$. In case 2 , link lengths and discharges are as follows: for $\Omega=5$ : (1) $1.437,2.065$, (2) 1.609 , 6.719 . (3) $1.802,16.685$, (4) $2.018,37.442$, (5) $2.26,80$; for $\Omega=2$ : (1) $1.779,3.165$, (2) $1.416,8.335$, (3) $1.416,13.505, \cdots$, (15) 1.416 , 75.545 , (16) $1.416,80$; for $\Omega=3$ : (1) $1.762,3.105$, (2) $1.566,8.662$, (3) $1.566,14.219$, (4) $1.566,19.776$, (5) $1.175,35.376$, (6) $1.175,50.976$, (7) $1.175,55.462$, (8) $1.175,71.062$, (9) $1.175,75.548$, (10) $1.175,80$. Total rates of energy expenditure are as follows: $\Omega=5,134 k ; \Omega=$ $2,187 k ; \Omega=3,153 k$.

the space in which the network will develop. This is certainly not the case for river networks which drain a space conditional on its shape by the very large scale features of the terrain. According to the third principle of optimal energy expenditure, the topological arrangement of the network elements in this space will be such as to lead to the minimization of the total rate of energy expenditure, $E$. It will be shown through an example that the third principle leads to drainage networks which obey Horton's laws of stream numbers and stream lengths with the values of $R_{b}$ and $R_{L}$ typically observed in nature.

A square grid is superimposed on a rectangular region of $30 \times 60$ grid units, the problem being to find the network which minimizes the function $E$. The optimization imple-
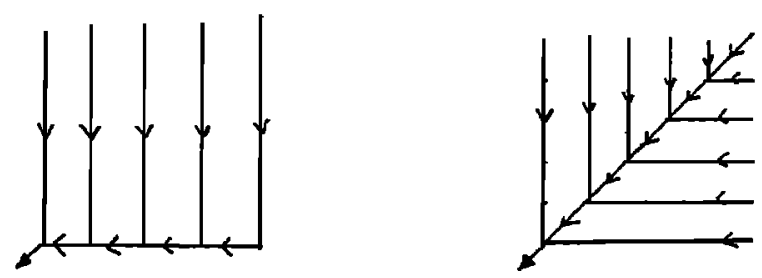

Fig. 3. Initial network configurations for the optimization procedure.

mented is similar to the strategy developed by Lin [1965] for the traveling salesman problem. It starts with an initial configuration which is perturbed by randomly changing the flow direction of a randomly chosen node among its eight surrounding neighbors under the constraint that the network should drain the whole area to a common outlet, no lakes being allowed inside the basin. The change on $E$ is computed between the new and the old configuration, and if it is negative, the new configuration is adopted as the base configuration and the process is iterated. On the other hand, if the change on $E$ is positive, the old configuration is perturbed again. The procedure leads to a network in which no improvement on $E$ appears after many perturbations. The entire process is repeated many times to obtain a set of networks with different minimal $E$. The configuration with the overall minimum $\boldsymbol{\Sigma}_{\boldsymbol{i}} \boldsymbol{P}_{\boldsymbol{i}}$ is selected as the solution to the problem. Although there is no assurance that the solution represents a global optimum, experience shows that the procedure is quite effective in obtaining solutions near the global optimum starting from an arbitrary initial configuration. Figure 3 shows examples of the initial configurations that were chosen, very far indeed from the appearance of natural drainage networks. Figure 4 shows an example of the obtained optimal configurations. Throughout the whole procedure stream channels are defined as those pixels with a cumulative drainage area greater than a support threshold which is taken as five pixels in these experiments. Although more research is needed in this area, it appears that the third principle of optimal energy expenditure leads to networks of striking Hortonian structure with $R_{b}$ and $R_{L}$ values as those observed in natural river basins. Moreover, it also leads to power law probability distributions of the discharge and energy dissipation throughout the population of links which have the same exponents as those found by RodriguezIturbe et al. [this issue] in natural basins. This topic will be described in detail in a different paper by the authors.

\section{SCALING IMPLICATIONS OF OPTIMAL ENERgy EXPENDITURE}

The rate of energy expenditure per unit area of channel, $\boldsymbol{P}_{1}$, may be written as

$$
P_{1}=\frac{\rho g Q S}{(w+2 d)}+K C_{f}^{m} \rho^{m} v^{2 m}
$$

Substituting for $w$ and $d$, the expressions obtained from the joint application of the two principles of energy expenditure, $d \propto Q^{0.5}$ and $w \propto Q^{0.5}$, one obtains (with constant velocity throughout the network)

$$
P_{1}=c_{1} Q^{0.5} S+c_{2}
$$




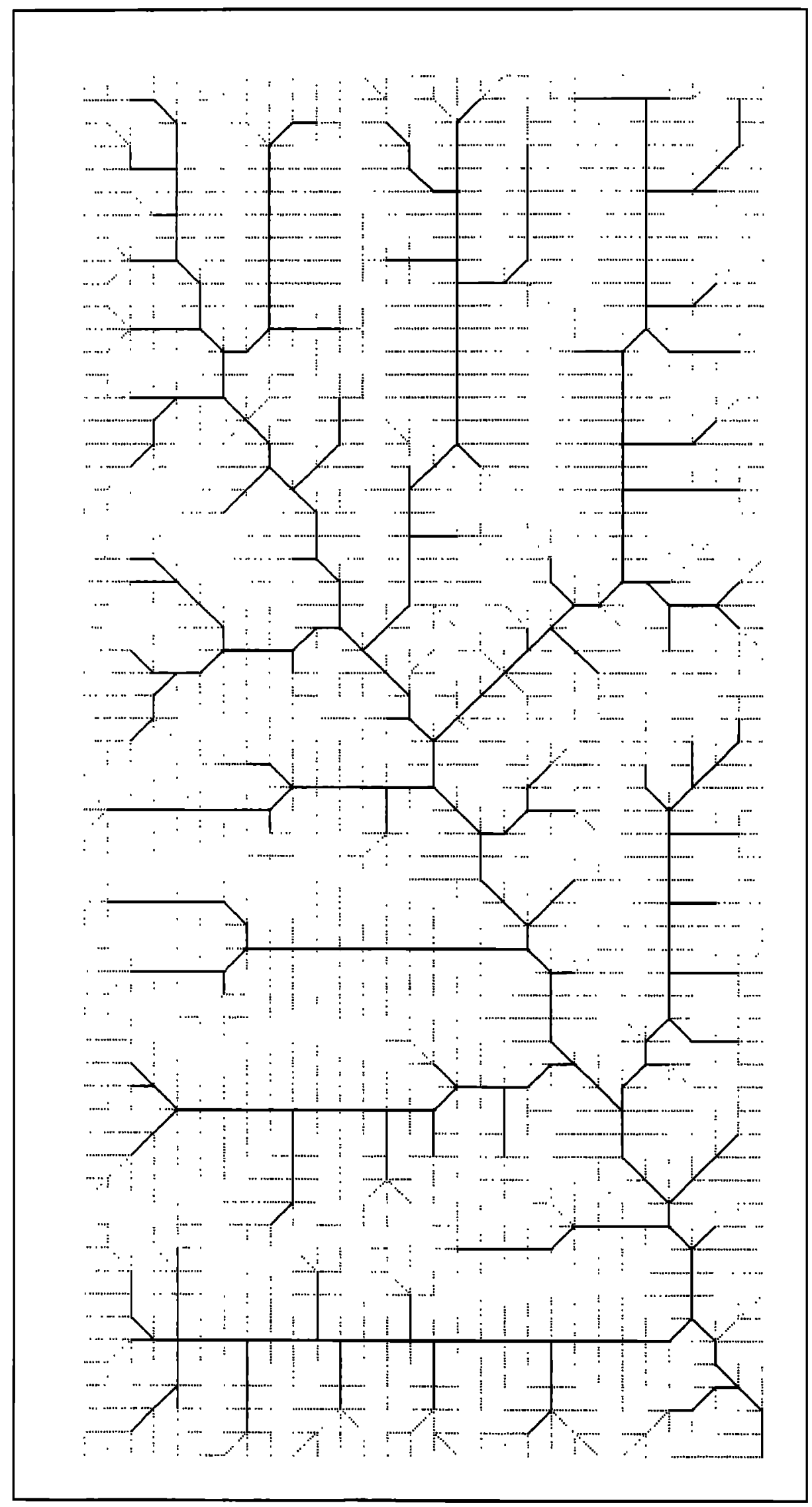

Fig. 4. Network with near-optimal minimum $E: N_{1}=83, N_{2}=16, N_{3}=4, N_{4}=1 ; R_{B}=4.36 ; L_{1}=2.92$, $\bar{L}_{2}=7.02, \bar{L}_{3}=17.05, \bar{L}_{4}=43.46 ; R_{L}=2.46$. 


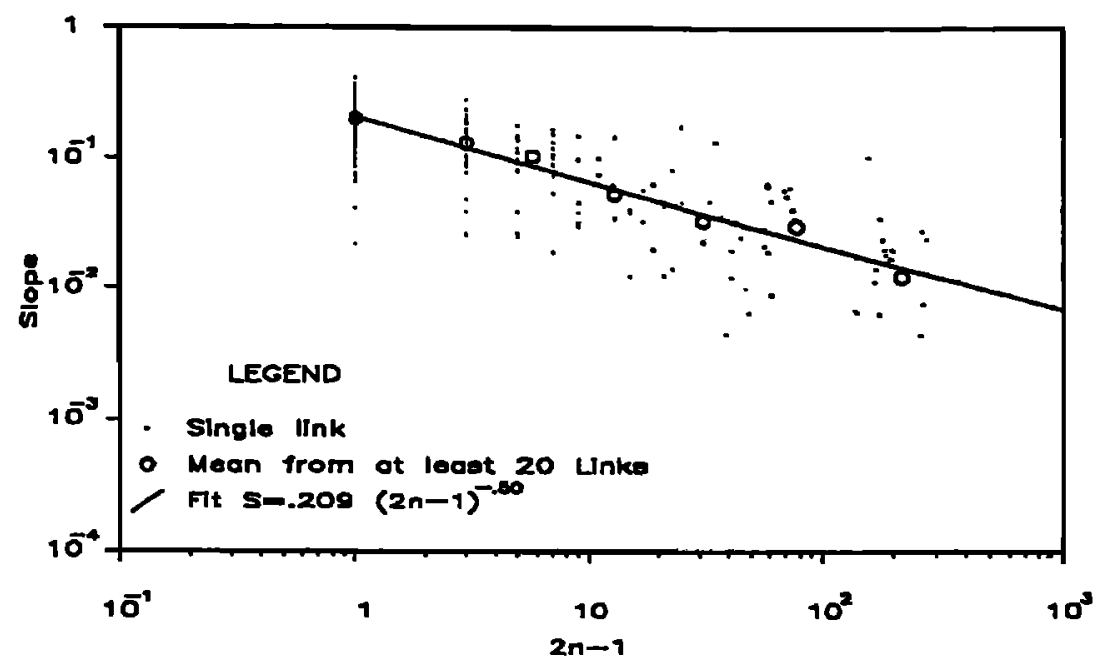

Fig. 5. Link slopes as function of magnitude for Big Creek, Idaho [from Tarboton et al., 1989].

For a given flow condition, $c_{1}$ and $c_{2}$ are constant throughout the network, and under the second principle $P_{1}$ is constant in all links. Thus

$$
Q^{0.5} S=\text { const }
$$

implying that the product of the slope times the square root of the discharge is a constant throughout the network when mean annual flow conditions exist everywhere in the basin. Under bank-full discharge conditions, this will 'still be valid with a different constant on the right-hand side.

Studies of the stable channel section of gravel rivers by Parker [1978] and Ikeda et al. [1988] have shown that the product $d S$ is only a function of the sediment characteristics; with $d \propto Q^{0.5}$ this agrees with the previous result. Leopold and Wolman [1957] report a large number of rivers in the United States and India that show $S Q^{0.44}=$ const. Leopold et al. [1964] also report the values for the exponent $z$ in the relation $S \propto Q^{z}$ for observations in the downstream direction. An average value of $z=-0.49$ was obtained for streams in the midwestern United States for both bank-full and mean annual flow conditions. Nevertheless, for ephemeral streams in semiarid regions, they quote an exponent closer to -1.0 . The above field data are probably quite unreliable for studying the relationship $S Q^{z}$. One needs to measure both the discharge and the slope along individual links. The identification of the network itself is not a trivial matter, and it is only recently through digital elevation maps (DEMs) that the network with the slopes of its links and their individual contributing areas has been objectively studied. DEMs consist of elevations in a rectangular grid with, usually, $30 \mathrm{~m}$ spacing. In the United States, grids with $30 \mathrm{~m}$ to a side are common. Each grid block is termed a pixel and streams are then usually defined as those pixels with cumulative drainage area greater than a support area threshold [O'Callaghan and Marks, 1984; Band, 1986].

Discharge measurements in every link of a network are not available and since the mean annual flow has been observed to be proportional to the drainage area in many regions of the world, area may then be used as a surrogate variable for discharge and $Q^{0.5} S=$ const becomes $A^{0.5} S=$ const. This relationship can be studied in detail using DEMs. The magnitude of a link, $n$, is defined as the number of sources upstream of the link. For topological reasons the total number of links draining through the outlet of a link of magnitude $n$ is $2 n-1$. The area draining directly to any link, $A^{*}$, varies randomly from link to link but does not depend on the magnitude. Thus the total area, $A(n)$, draining through a link of magnitude $n$ is itself a random variable,

$$
A(n)=\sum_{i=1}^{2 n-1} A_{i}^{*}
$$

Thus rather than considering the energy expenditure per unit area of channel as a constant anywhere in the network, it is now considered as a random variable, $\xi$, whose expected value is the same throughout the network. This is expressed as

$$
Q^{0.5}(n) S(n)=\xi(n)
$$

where $E[\xi(n)]=$ const. Using $A(n)$ as a surrogate of $Q(n)$, (17) yields in first-order analysis

$$
E[S(n)]=\text { const }(2 n-1)^{-0.5}
$$

Thus the principles of optimal energy expenditure lead to the scaling of the mean link slopes as a function of areas or their surrogate variables, magnitudes. The basic scaling is in terms of discharges, but with discharges proportional to areas (18) is a natural consequence. The scaling of (18) is precisely of the type suggested by Gupta and Waymire [1989] and the value of -0.5 is the one found in the analysis of drainage networks through DEMs by Tarboton et al. [1989], an example of which is shown in Figure 5. From first-order analysis of (17) the variance of link slopes may be obtained as

$$
\operatorname{Var}[S(n)] \propto(2 n-1)^{-1} \operatorname{Var}[\xi(n)]
$$

Flint [1974] and Tarboton et al. [1989] have found that Var $[S(n)]$ scales as $(2 n-1)^{-0.5}$ which then implies that

$$
\operatorname{Var}[\xi(n)] \propto(2 n-1)^{0.5}
$$

Figure 6 shows an example of the mean and variance of $A^{0.5}(n) S(n)$ as functions of magnitude for the Racoon River 


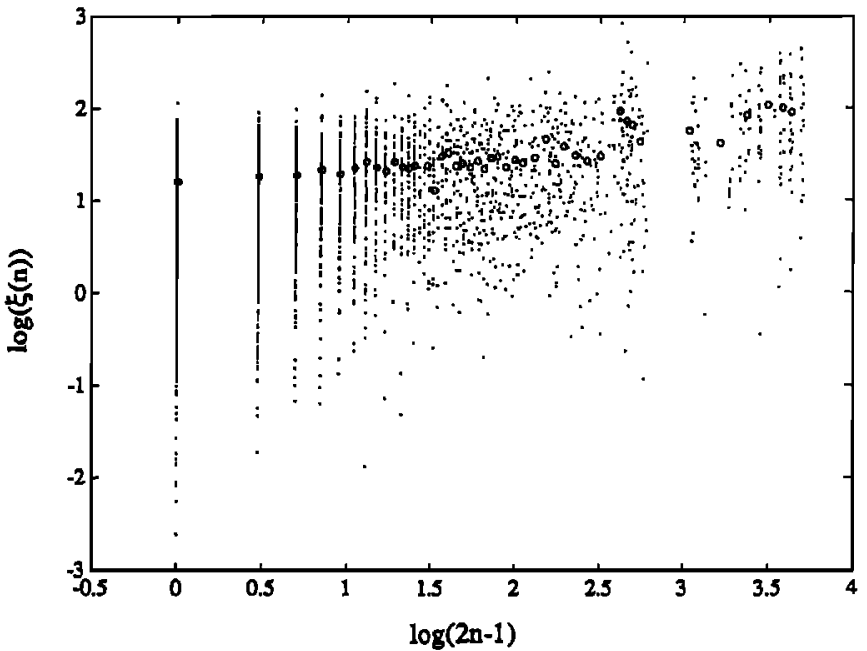

Fig. 6. Mean and variance of $\xi(n)$ versus $(2 n-1)$ for the Racoon River basin in Pennsylvania.

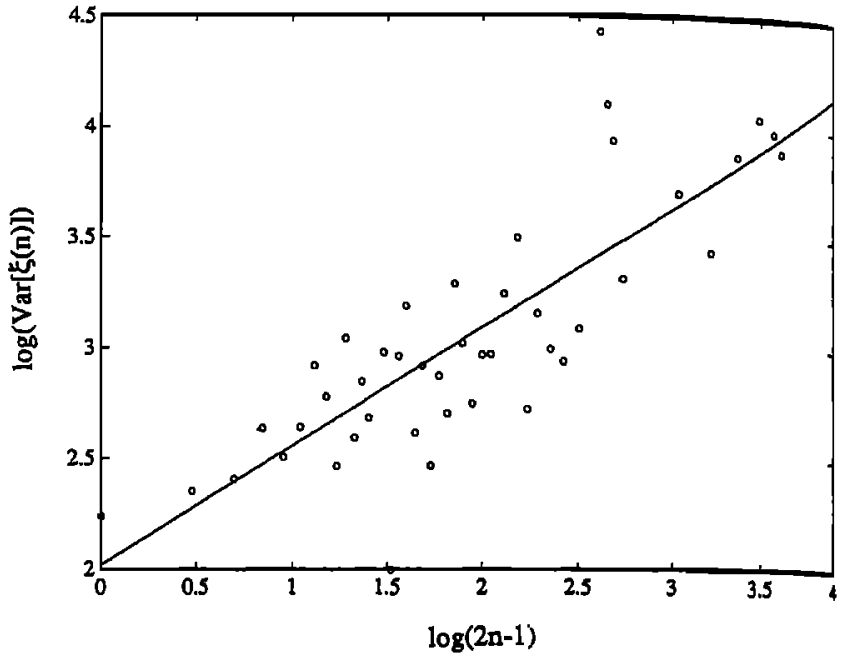

basin in Pennsylvania. Figure 6 is constructed grouping links according to magnitude so that there are at least 25 links with identifiable slopes in every interval of the histogram. Due to the $1-\mathrm{m}$ vertical resolution of DEM there are links whose altitude drop and the corresponding slopes cannot be identified. In these cases a random altitude drop between 0 and 1 $m$ is assigned to the link and the corresponding slope is then computed as the ratio of altitude drop and link length. The lines in Figure 6 were fitted by least squares through the whole set of points. It is observed that the mean is independent of magnitude, as predicted by the second principle of optimal energy expenditure, and the variance is proportional to $(2 n-1)^{0.5}$. This is an indication of multiscaling in $S(n)$ where there is not just one scaling relation determining all the moments of the process but rather changes of scale affect different moments with different scaling laws.

The multiscaling character of $S(n)$ points toward the fact that variance of the random variable whose expected value is the same throughout the network increases proportionally to the average travel time for the flow to reach any site in the system. The reason for this lies in the nature of the energy dissipation along any flow path in the network. Energy is spent along a succession of pools and riffles analogous to a diffusion of the energy along the flow path or equivalently to a random walk in the altitude space through which small drops of random height occur randomly along the flow path [Tarboton et al., 1989]. From the point of view of the optimal operation of the network, it is desirable that the expected value of the energy spent per unit area of channel be the same everywhere in the basin but the variance of such energy expenditure, similar to that in a diffusion process, will be proportional to the length of the path or equivalent to the average travel time for the flow to reach a particular link from all its tributary links. The average length of flow path at any point may be considered proportional to the square root of the total area draining at the point and thus $\operatorname{Var}[\xi(n)] \propto$ $[(2 n-1)]^{0.5}$.

The spatial structure of runoff production is intimately linked to the scaling of the drainage network. Equation (17) may be written as

$$
\begin{aligned}
{\left[i_{1} \beta_{1} A_{1}^{*}\right.} & +i_{2} \beta_{2} A_{2}^{*}+\cdots \\
& \left.+i_{(2 n-1)} \beta_{(2 n-1)} A_{(2 n-1)}^{*}\right]^{0.5} S(n)=\xi(n)
\end{aligned}
$$

where $i$ is the mean annual rainfall input, and $\beta$ is the mean annual runoff coefficient of the area draining into the individual links upstream of a link of magnitude $n$. The fact that $E[S(n)] \propto(2 n-1)^{-0.5}$ and $E[\xi(n)]=$ const indicates that in first-order analysis basins tend to be organized so that the expected value of annual runoff production per unit area, $i_{r} \beta$, remains approximately the same throughout the basin.

\section{Conclusions}

The combination of three principles of energy expenditure results in a unified picture of the most important empirical findings related to the dynamics of the three-dimensional structure of drainage networks. Among them are these: (I) the velocity of the flow for a representative discharge tends to be constant throughout the network; (2) the depth of flow is proportional to the square root of the discharge, the constant of proportionality being the same everywhere in the network; (3) in the downstream direction the channel width varies proportionally to the square root of the discharge; and (4) the mean value of the slopes of links with magnitude $n$ scale proportionally to $(2 n-1)^{-0.5}$. The degree of bifurcation of the network and the relative length of its elements as measured by the bifurcation and length ratios usually found in nature in the narrow ranges $3 \leq R_{b} \leq S$ and $1.5 \leq R_{L} \leq$ 3.5 may also be interpreted as consequences of the three principles of energy expenditure. The multiscaling characteristics observed in the three-dimensional structure of the network are also related to the manner that the expenditure of energy takes place in the drainage basin.

Acknowledgments. During the research leading to this paper, I. Rodriguez-Iturbe was at the Institute of Hydraulic Research of the University of Iowa, Iowa City. The support of the Iowa Economic Development Commission is gratefully acknowledged. At MIT this work was supported by the U.S. Army Research Office, agreement DAAL03-89-K-0151. In Italy funds were granted by the projoct 
MURST $40 \%$ "Ferromeris di Trasporto nel Ciclo Idrologica." The views, opinions and/or findings contained in this report are those of the authors and should not be construed as an official Department of the Army position, policy, or decision, unless so designated by other documentation.

\section{REFERENCES}

Band, L. E., Topographic partition of watersheds with digital elevation models, Water Resour. Res., 22(1), 15-24, 1986.

Brush, L. M., Jr., Drainage basins, channels and flow characteristics of selected streams in central Pennsylvania, U.S. Geol. Surv. Prof. Pap., 282-F, 1961 .

Flint, J. J., Stream gradient as a function of order, magnitude, and discharge, Water Resour. Res., 10(5), 969-973, 1974.

Gupta, V. K., and E. Waymire, Statistical self-similarity in river networks parameterized by elevation, Water Resour. Res., 25(3), 463-476, 1989.

Horton, R. E., Erosional development of streams and their drainage basins; hydrophysical approach to quantitative morphology, Geol. Soc. Am. Bull., 56, 275-370, 1945.

Howard, A. D., Simulation of stream networks by headward growth and branching, Geogr. Anal., 3, 29-50, 1971.

Howàrd, A. D., Theoretical model of optimal drainage networks, Water Resour. Res., 26(9), 2107-2117, 1990.

Ikeda, S., G. Parker, and Y. Kimura, Stable width and depth of straight gravel rivers with heterogeneous bed materials, Water Resour. Res., 24, 713-722, 1988.

La Barbera, P., and R. Rosso, The fractal geometry of river networks (abstract), Eos Trans. AGU, 68, 1276, 1987.

Leopold, L. B., and W. L. Langbein, The concept of entropy in landscape evolution, U.S. Geol. Surv. Prof. Pap., 500-A, 1962.

Leopold, L. B., and T. Maddock, Jr., The hydraulic geometry of stream channels and some physiographic implications, U.S. Geol. Surv. Prof. Pap., 252, 1953.

Leopold, L. B., and M. G. Wolman, River channel patterns: Braided, meandering, and straight, U.S. Geol. Surv: Prof. Pap., 282- $B, 1957$.

Leopold, L. B., M. G. Wolman, and J. P. Miller, Fluvial Process in Geomorphology, W. H. Freeman, New York, 1964.

Lin, S., Computer solutions for the traveling salesman problem, Bel Syst. Tech. J., 44, 2245-2269, 1965.

Murray, C. D., The physiological principle of minimum work, I, The vascular system and the cost of blood volume, Proc. Natl. Acad. Sci. U.S.A., 12, 207-214, 1926.

O'Callaghan, J. F., and D. M. Marks, The extraction of drainage networks from digital elevation data, Comput. Vision Graphics Image Process., 28, 323-344, 1984.
Parker, G., Self-formed straight rivers with equilibrium banks and mobile bed, 2, The gravel river, J. Fluid Mech., 89, 127-146, 1978.

Pilgrim, P. H., Isochrones of travel time and distribution of flood storage from a tracer study on a small watershed, Water Resour. Res., 13(3), 587-595, 1977.

Rodríguez-Iturbe, I., E. J. Ijiász-Vásquez, R. L. Bras, and D. G. Tarboton, Power law distributions of discharge mass and energy in river basins, Water Resour. Res., this issue.

Roy, A. G., Optimal angular geometry models for river branching, Geogr. Anal., 15, 87-96, 1983.

Sherman, T. F., On connecting large vessels to small, J. Gen. Physiol., 78(4), 431-453, 1981.

Shreve, R. L., Statistical law of stream numbers, J. Geol., 74, 17-37, 1966.

Shreve, R. L., Stream lengths and basin areas in topologically random channel networks, J. Geol., 77, 397-414, 1969.

Stevens, P. S., Patterns in Nature, Little, Brown, Boston, Mass., 1974.

Tarboton, D. G., R. L. Bras, and I. Rodríguez-Iturbe, The fractal nature of river networks, Water Resour. Res., 24(8), 1317-1322, 1988.

Tarboton, D. G., R. L. Bras, and I. Rodríguez-Iturbe, Scaling and elevation in river networks, Water Resour. Res., 25(9), 20372051, 1989.

Uylings, H. B. M., Optimization of diameters and bifurcation angles in lung and vascular tree structures, Bull. Math. Biol., 39, $509-520,1977$.

Woldenberg, M. J., and K. Horsfield, Relation of branching angles to optimality for four cost principles, J. Theor. Biol., I22, 204, 1986.

Wolman, M. G., The natural channel of Brandywine Creek, Pennsylvania, U.S., U.S. Geol. Surv. Prof. Pap., 271, 56 pp., 1955.

Yang, C. T., Potential energy and stream morphology, Water Resour. Res., 7(2), 311-322, 1971.

R. L. Bras and E. Ijjász-Vásquez, Ralph M. Parsons Laboratory, Massachusetts Institute of Technology, Cambridge, MA 02139. A. Marani and R. Rigon, Universita di Venezia, Dorsoduro 2137, Venezia, Italy.

A. Rinaldo, Universita di Trento, Mesiano di Provo, I-38050 Trento, Italy.

I. Rodríguez-Iturbe, Instituto Internacional de Estudios Avanzados, P.O. Box 17606, Parque Central, Caracas, Venezuela.

(Received May 20, 1991;

revised November 13,1991; accepted December 5, 1991.) 